\title{
Elemental Distribution Analysis of Core/Shell Nanocrystals with STEM/EDX
}

\author{
Jacob T. Held ${ }^{1}$, Katharine Hunter ${ }^{2}$, Uwe R. Kortshagen ${ }^{2}$ and K. Andre Mkhoyan ${ }^{1}$ \\ ${ }^{1 .}$ Department of Chemical Engineering \& Materials Science, University of Minnesota, Minneapolis, \\ MN. \\ 2. Department of Mechanical Engineering, University of Minnesota, Minneapolis, MN.
}

Semiconductor quantum dots exhibit many interesting and useful size-dependent optoelectronic properties. ${ }^{1}$ Recently, considerable effort has been invested in developing reliable methods for producing core/shell quantum dots. By manipulating the band alignment and strain between the core and shell, new avenues of optoelectronic tunability are being explored., ${ }^{2,3}$ However, the properties of these systems are sensitive to the interface between the core and shell materials and characterizing this interface remains a significant challenge. ${ }^{4}$ In this study, we build upon previous work ${ }^{5}$ and demonstrate a method for quantifying the radial distribution of elements in spherical core/shell nanocrystals by fitting simulated distributions to radially-averaged STEM/EDX data. This approach yields quantitative measurements of interface broadening, outer surface roughness, and core/shell composition.

Plasma-grown Ge/Si core/shell nanocrystals with well-controlled core and shell thickness ${ }^{6}$ were used as an ideal test case for demonstration of this technique (Figure 1). The nanocrystals were directly deposited onto holey/thin double carbon grids and immediately transferred into the microscope under Ar. All particles were imaged in an FEI-Titan G2 60-300 equipped with a Super-X EDX system running at $60 \mathrm{kV}$ with a 25 mrad convergence angle, $125 \mathrm{pA}$ beam current, and a 68 mrad HAADF inner angle. EDX spectrum images were acquired with frame-by-frame drift correction with a dwell time of $3 \mu$ s/pixel and a pixel resolution of $0.03 \mathrm{~nm} /$ pixel. The resulting spectrum images (Figure 2a-d) were radially averaged around the centroid of a fit ellipse (major/minor axes within <5\%) to produce 1D datasets for further analysis.

An error function based continuum model was developed to describe both the interface broadening and the outer surface roughness as well as to account for residual core material in the shell due to synthesis conditions. The spherical radial distributions were numerically projected down the cylindrical $\mathrm{Z}$ axis and convoluted with a Gaussian probe function to account for probe spread, producing a fit to the experimental EDX data. The simulation parameters (core and shell radii, interface broadening, surface roughness, and shell composition) were then optimized by minimizing the sum-squared error of the fit. Uncertainty in the fit values was defined as the maximum change in each parameter that was necessary to increase the fitting error by $5 \%$. The results of applying this fitting to a Ge/Si core/shell nanocrystal are shown in Figure 2. Because this approach requires only a single STEM/EDX spectrum image for each nanocrystal and uses all of the EDX counts acquired from a 2D map of the crystal through radial averaging, this method yields a much higher average signal to noise ratio than traditional line profiles acquired with the same electron dose per beam position. While the methods presented in this work approximate the nanocrystals as spheres, which is appropriate for the samples studied, analogous fitting methods could be developed for other known geometries [7].

\section{References:}

[1] K. Bourzac. Nature 493 (2013) p. 283.

[2] B. O. Dabbousi, et al. J. Phys. Chem B 101 (1997) p. 9463.

[3] S. Kim, et al. J. Am. Chem. Soc. 125 (2003) p. 11466. 
[4] N. Tschirner, et al. Chem. Mater. 24 (2012) p. 311.

[5] J. T. Held, et al. Microscopy and Microanalysis, 22(S3) (2016), p. 128.

[6] K. I. Hunter, et al. ACS Appl. Mater. Interfaces. doi: 10.1021/acsami.6b16170

[7] This work was supported primarily by NSF through the University of Minnesota MRSEC under Award Number DMR-1420013.
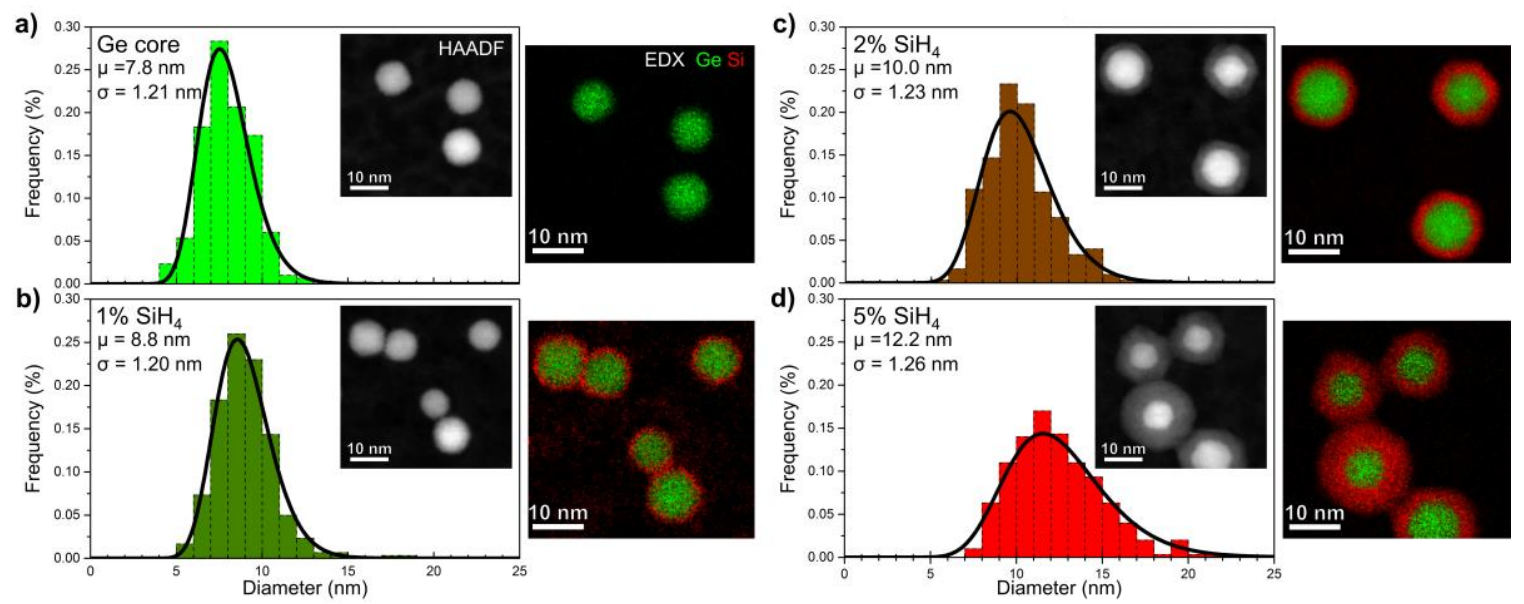

Figure 1. Size distributions, HAADF images (inset), and composite EDX maps of Ge (green) and Si (red) plasma-grown core/shell nanocrystals, synthesized with $0,1,2$, and $5 \%$ concentrations of $\mathrm{SiH}_{4}$ in the shell growth gas feed. Size distributions were measured from HAADF-STEM images of 300 nanocrystals per sample and the geometric mean and standard deviation were obtained by fitting each histogram with a log-normal distribution. Figure adapted from ref. 7.

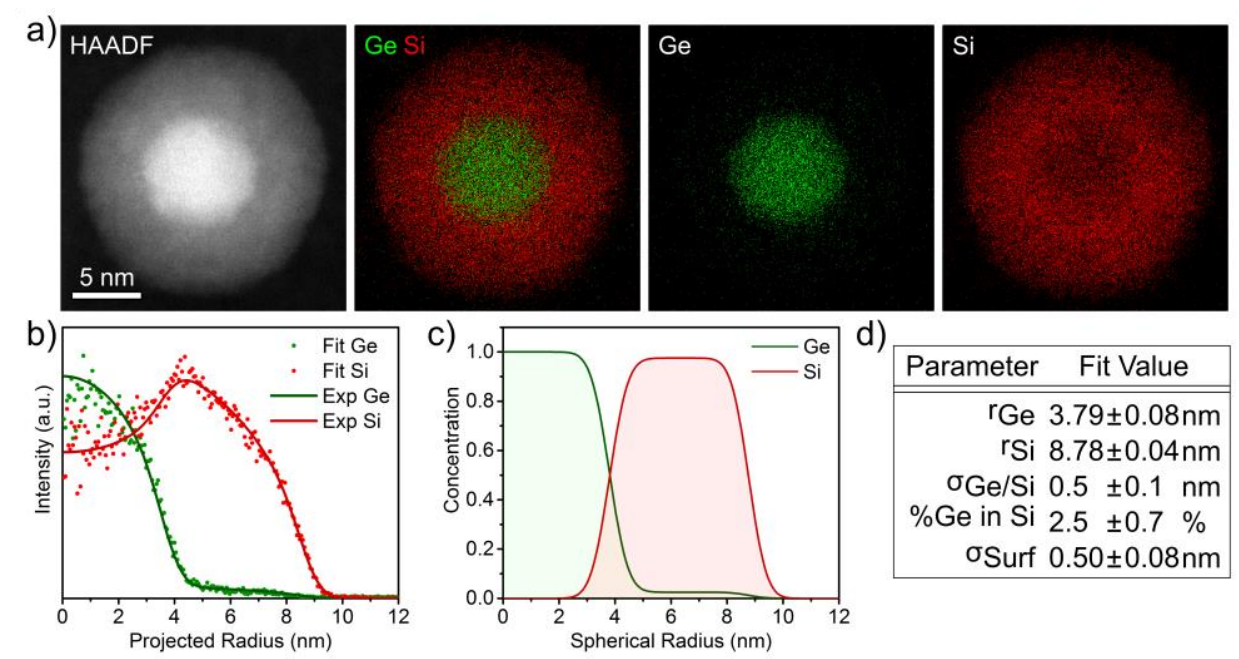

Figure 2. Ge/Si core/shell particle analysis. (a) Experimental spectrum image dataset including a raw HAADF-STEM image, composite Ge (green) and Si (red) EDX map, and separate Ge and Si maps. (b) Radially-averaged EDX data and corresponding projected fit profiles (rescaled for figure). (c) Spherical elemental distribution of Si and Ge of the best-fit model. (d) Optimized values for each fitting parameter. Not shown: average probe $\mathrm{fwhm}=0.18 \mathrm{~nm}$. 